\title{
A Beam-Steering Antenna Array Using Injection Locked Coupled Oscillators With Self-Tuning of Oscillator Free-Running Frequencies
}

\author{
Sheng-Hong Yan, Student Member, IEEE, and Tah-Hsiung Chu, Member, IEEE
}

\begin{abstract}
The analysis and experimental results of an antenna array using injection locked coupled oscillators with self-tuning of oscillator free-running frequencies are presented. With the use of coupled type-II phase locked loops for tuning oscillator free-running frequencies and an external injection signal for stabilizing the array operating frequency, this antenna array can steer its beam through a single control voltage and hold its output frequency at the injection signal frequency in operation. In addition, its beam-pointing error arising from phase errors in coupled oscillators can be reduced and the array works well over a certain frequency band. Phase dynamics and stability are studied and experimentally verified. Experimental results of a three-element injection locked coupled oscillator array show that its uniform phase progression ranges between $-16^{\circ}$ and $52^{\circ}$, and the phase errors are less than $5^{\circ}$ at $2.7 \mathrm{GHz}$. The operation bandwidth is shown from 2.68-2.72 GHz. By loading the injection locked coupled oscillator array with rectangular patch antennas, the beam-steering radiation characteristics are measured at various control voltages.
\end{abstract}

Index Terms-Coupled oscillators, injection locked oscillators, phase locked loops, stability.

\section{INTRODUCTION}

B EAM-STEERING phased antenna arrays find many applications in microwave radar and communication systems. A conventional phased antenna array uses a power distribution network, phase shifters, and control signals to provide its output signals with the desired phase distribution to its radiating antennas. This approach, however, utilizes components such as a feeding network and variable phase shifters not only bulky but also lossy, thus increasing design complexity. In addition, beam steering is designed for single frequency operation due to the limitation of phase shifters.

Coupled oscillator array (COA) is a prominent alternative to offer its output signals with the required phase distribution to the connected antennas without the use of phase shifters [1]-[7] or with the use of a single phase shifter [8]. The operation of phase distribution control is based on the injection locking mechanism of oscillators [9]-[11]. For a linear COA in a phased array,

Manuscript received April 11, 2007; revised February 5, 2008. Published September 4, 2008 (projected). This work was supported by the National Science Council of the R.O.C. under Grants NSC 95-2221-E002-086-MY3 and NSC 96-2752-E002-004-PAE.

The authors are with the Graduate Institute of Communication Engineering, National Taiwan University, Taipei, Taiwan, R.O.C. (e-mail: thc@ew.ee.ntu. edu.tw).

Digital Object Identifier 10.1109/TAP.2008.928770 nearest-neighboring coupling is the most popular structure, and its output signals with a uniform progressive phase distribution is often taken into consideration, meaning that the phase difference between the output signals of successive elements is a constant. The behavior of such an array can be expressed by a set of nonlinear differential equations [1], [12]-[14]. In theory, one can adjust the free-running frequencies of the two outermost oscillators in opposite directions through two control signals to yield a uniform phase progression ranging between $-90^{\circ}$ and $90^{\circ}$ for beam-steering applications. However, this method can be adopted only at the single design frequency. As the operation frequency is changed, all the oscillator free-running frequencies should be tuned to acquire the desired beam pattern.

Circuit fabrication inconsistencies, however, lead to fluctuations in the free-running frequencies and amplitudes of the oscillators. The amplitudes of the two end oscillators of a COA also change from their original values when they are adjusted in opposite directions. These fluctuations then cause phase errors and the consequent beam-pointing error in a COA-based phased antenna array [15]. The common frequency of the oscillator array might also deviate from the design frequency because of these fluctuations. In [16], an approach to optimize the oscillator fabrication is proposed to diminish the impact of such fluctuations. Nevertheless, the individual bias at each oscillator allows one to establish the required free-running frequency distribution for a given uniform phase progression through bias tuning. This method can solve the problems resulting from these fluctuations as well.

Phase locked loop (PLL) is a well-known approach to stabilize the oscillating frequency of an oscillator. In [17]-[20], the studies focus on using a coupled type-I PLL array to synchronize the oscillators instead of the injection locking approach used in a COA. The type of a PLL is the number of poles in its open-loop transfer function $G(s)$ that are at the origin. This structure has a wider locking range than a COA and gives uniform progressive phase control. However, it also suffers from phase errors and the variation in the common frequency, which result from the fluctuations in the free-running frequencies of the oscillators. Tuning the free-running frequency of each oscillator can settle these problems.

In this paper, the design of an antenna array using an injection locked coupled oscillator array (ILCOA) with self-tuning of oscillator free-running frequencies is presented. With the use of coupled type-II PLLs for tuning the oscillators and an external injection signal for stabilizing the array operating frequency, the array can steer its beam through a single control 


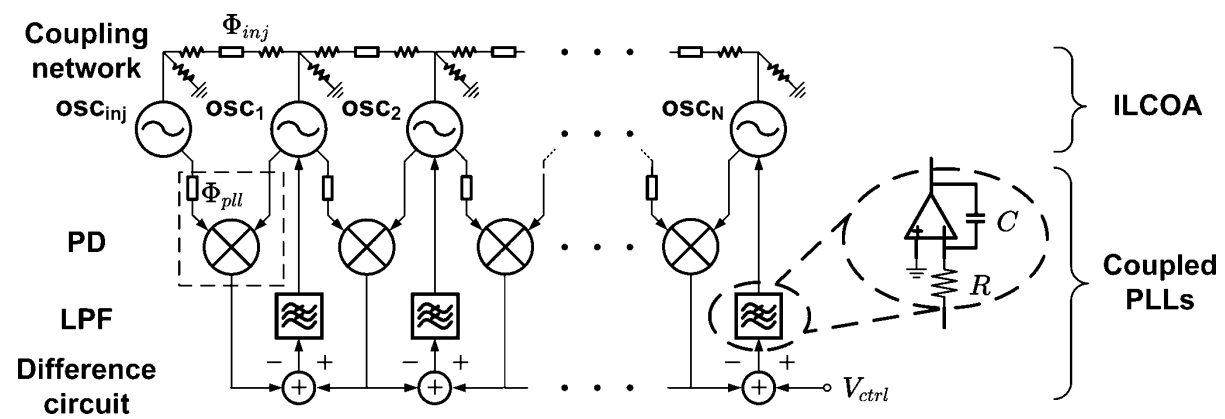

Fig. 1. Configuration of an $N$-element ILCOA integrated with coupled type-II PLLs.

voltage and hold its output frequency at the injection signal frequency. In addition, its beam-pointing error arising from the phase errors in coupled oscillators can be reduced and the array works well over a certain frequency band. In contrast, controlling the beam-scanning angle and the operation frequency of a conventional COA-based antenna array may require significant time and efforts when fluctuations in the free-running frequencies and amplitudes of the oscillators are considered. In the following, the theory and experimental results are described in Sections II and III in detail. Some discussions and conclusions are given in Section IV. In the Appendix, the stability analysis shows that the uniform progressive phase distribution is the only one stable solution of the dynamic equations.

\section{THEORY}

Fig. 1 illustrates the configuration of an $N$-element ILCOA integrated with coupled type-II PLLs. The ILCOA consists of $N+1$ oscillators. The left-end oscillator represents an external injection signal, and the others form a COA through a resistive coupling network. Within the array, a type-II PLL consists of a phase detector (PD), a difference circuit, and a low pass filter (LPF) of an inverting integrator. While the oscillators are coupled through the resistive coupling network, their signals are also fed to the adjacent PDs to measure the phase difference between the output signals of successive oscillators. The phase shift $\Phi_{\mathrm{pll}}=-90^{\circ}$ makes the PD produce an output signal to the neighboring difference circuits as the sine function of the measured phase difference. The difference circuit then produces an output signal to the tuning port of the connected oscillator through the LPF to tune the oscillator free-running frequency.

The phase dynamics for this $N$-element ILCOA integrated with coupled type-II PLLs can be given as [9], [12], [18], [21]

$$
\begin{aligned}
\frac{d}{d t} \theta_{\mathrm{inj}}= & \omega_{\mathrm{inj}} \\
\frac{d}{d t} \theta_{1}= & \omega_{\mathrm{fr}, 1}+\frac{\epsilon \omega_{\mathrm{fr}, 1}}{2 Q_{1}} \\
\times & {\left[\frac{A_{\mathrm{inj}}}{A_{1}} \sin \left(\theta_{\mathrm{inj}}-\theta_{1}+\Phi_{\mathrm{inj}}\right)+\frac{A_{2}}{A_{1}} \sin \left(\theta_{2}-\theta_{1}+\Phi_{\mathrm{inj}}\right)\right] } \\
& +K_{v} K_{\mathrm{pd}}\left[-\cos \left(\theta_{\mathrm{inj}}-\theta_{1}+\Phi_{\mathrm{pll}}\right)\right. \\
& \left.+\cos \left(\theta_{2}-\theta_{1}-\Phi_{\mathrm{pll}}\right)\right] * f(t)
\end{aligned}
$$

$$
\begin{aligned}
\frac{d}{d t} \theta_{i}= & \omega_{\mathrm{fr}, i}+\frac{\epsilon \omega_{\mathrm{fr}, i}}{2 Q_{i}} \sum_{\substack{j=i-1 \\
j \neq i}}^{i+1} \frac{A_{j}}{A_{i}} \sin \left(\theta_{j}-\theta_{i}+\Phi_{\mathrm{inj}}\right) \\
+ & K_{v} K_{\mathrm{pd}}\left[\sum_{\substack{j=i-1 \\
j \neq i}}^{i+1}(j-i) \cos \left(\theta_{j}-\theta_{i}-(j-i) \Phi_{\mathrm{pll}}\right)\right] * f(t) \\
\frac{d}{d t} \theta_{N}= & \omega_{\mathrm{fr}, N}+\frac{\epsilon \omega_{\mathrm{fr}, N}}{2 Q_{N}} \sin \left(\theta_{N-1}-\theta_{N}+\Phi_{\mathrm{inj}}\right) \\
& +K_{v}\left[-K_{\mathrm{pd}} \cos \left(\theta_{N-1}-\theta_{N}\right.\right. \\
& \left.\left.+\Phi_{\mathrm{pll}}\right)+V_{\mathrm{ctrl}}\right] * f(t)
\end{aligned}
$$

for $i=2, \ldots, N-1 . \theta_{i}, A_{i}, \theta_{\mathrm{inj}}$, and $A_{\mathrm{inj}}$ are the phase and amplitude terms of the output signals of the $i$ th oscillator and the injection source, respectively. $\omega_{\mathrm{fr}, i}$ is the free-running frequency of the $i$ th oscillator. $\omega_{\text {inj }}$ is the frequency of the injection signal. $\epsilon$ and $\Phi_{\text {inj }}$ are the coupling strength and phase to account for the resistive coupling network. $Q_{i}$ is the resonator quality factor of the $i$ th oscillator. $K_{v}$ is the oscillator tuning sensitivity. $K_{\mathrm{pd}}$ is the PD conversion gain. $\Phi_{\mathrm{pll}}$ is the phase shift used to determine the PLL stable equilibrium point. $f(t)=-u(t) / R C$ is the impulse response of the LPF where $R$ and $C$ are the resistance and capacitance of the filter and $u(t)$ is a unit step function. $*$ is the convolution operator. $V_{\text {ctrl }}$ is the control voltage to determine the phase difference.

When the array reaches synchronization

$$
\begin{aligned}
\Delta \theta_{0} & =\theta_{\mathrm{inj}}-\theta_{1} \\
\Delta \theta_{i} & =\theta_{i}-\theta_{i+1}
\end{aligned}
$$

and $d \theta_{i} / d t=\omega_{\text {inj }}$ are constants. Due to the characteristics of inverting integrators, (1) implies

$$
\begin{aligned}
& \cos \left(\Delta \theta_{i-1}+\Phi_{\mathrm{pll}}\right)=\cos \left(\Delta \theta_{i}+\Phi_{\mathrm{pll}}\right) \\
& K_{\mathrm{pd}} \cos \left(\Delta \theta_{N-1}+\Phi_{\mathrm{pll}}\right)-V_{\mathrm{ctrl}}=0
\end{aligned}
$$

at that time. In other words

$$
\begin{aligned}
\left|\Delta \theta_{0}+\Phi_{\mathrm{pll}}\right| & =\left|\Delta \theta_{1}+\Phi_{\mathrm{pll}}\right|=\cdots=\left|\Delta \theta_{N-1}+\Phi_{\mathrm{pll}}\right| \\
& =\cos ^{-1}\left(\frac{V_{\mathrm{ctrl}}}{K_{\mathrm{pd}}}\right) .
\end{aligned}
$$


Therefore, the circuit shown in Fig. 1 has $2^{N}$ possible phase distributions. Letting $\Phi_{\mathrm{inj}}=-2 \pi$ and $\Phi_{\mathrm{pll}}=-\pi / 2$, the stable mode of (1) is

$$
\Delta \theta_{0}=\Delta \theta_{1}=\cdots=\Delta \theta_{N-1}=\sin ^{-1}\left(\frac{V_{\text {ctrl }}}{K_{\mathrm{pd}}}\right)
$$

which is the uniform progressive phase distribution. Note $V_{\text {ctrl }}$ is the only one control voltage applied to determine the phase progression value. However, there are two control voltages applied at the two outermost oscillators to adjust their free-running frequencies in a conventional COA [1]-[7], and $N$ tuning voltages might be needed to tune all the oscillators because of the fluctuations described above. From (1) and (7), the developed oscillator array shows that it can accurately and conveniently provide its output signals with a uniform progressive phase distribution. The phase difference is an inverse sine function of $V_{\text {ctrl }} / K_{\text {pd }}$ in spite of the fluctuations described above. In addition, the array can hold its output frequency stably at the injection signal frequency in operation. Moreover, the proposed array can work well as the injection signal frequency is varied within the injection signal locking range for multifrequency operation.

For an oscillator integrated with a type-I PLL, the phase difference is [17]

$$
\Delta \theta=\sin ^{-1}\left(\frac{V_{\text {tune }}+V_{\text {ctrl }}}{K_{\mathrm{pd}}}\right)
$$

where $K_{\mathrm{pd}}$ is the PD conversion gain. $V_{\text {tune }}$ is the voltage applied to the oscillator to make its free-running frequency be equal to the reference signal frequency. $V_{\text {ctrl }}$ is the control voltage to determine the phase difference. By comparing (7) when $N$ is equal to 1 with (8), one can find that if the oscillator free-running frequency deviates from the design frequency, the proposed circuit will automatically tune the oscillator free-running frequency and $\Delta \theta$ is determined only by $V_{\text {ctrl }}$.

The behavior of coupled oscillators depends on the coupling network. Recent studies have accurately predicted the performance of coupled oscillators for not only weak and strong coupling but also narrow-band and broadband coupling networks [7], [12], [18], [22], [23]. From (6), one can see that when the array reaches synchronization, there are $2^{N}$ possible phase distributions which satisfy (1) on the condition that the phase variable $\theta_{\text {inj }}$ is assigned. However, not all of these solutions are stable. For a phased antenna array to be a practical means of beam-steering radiation, the associated solution must be the stable state of the differential equations. In other words, any perturbation to give deviation from the desired phase distribution must rapidly decay to zero. The Appendix will give the stability analysis of the developed oscillator array with weak coupling through a broadband coupling network.

\section{EXPERIMENTAL VERIFICATION}

In this section, the detailed circuit design of an $N$-element ILCOA integrated with coupled type-II PLLs will be presented.

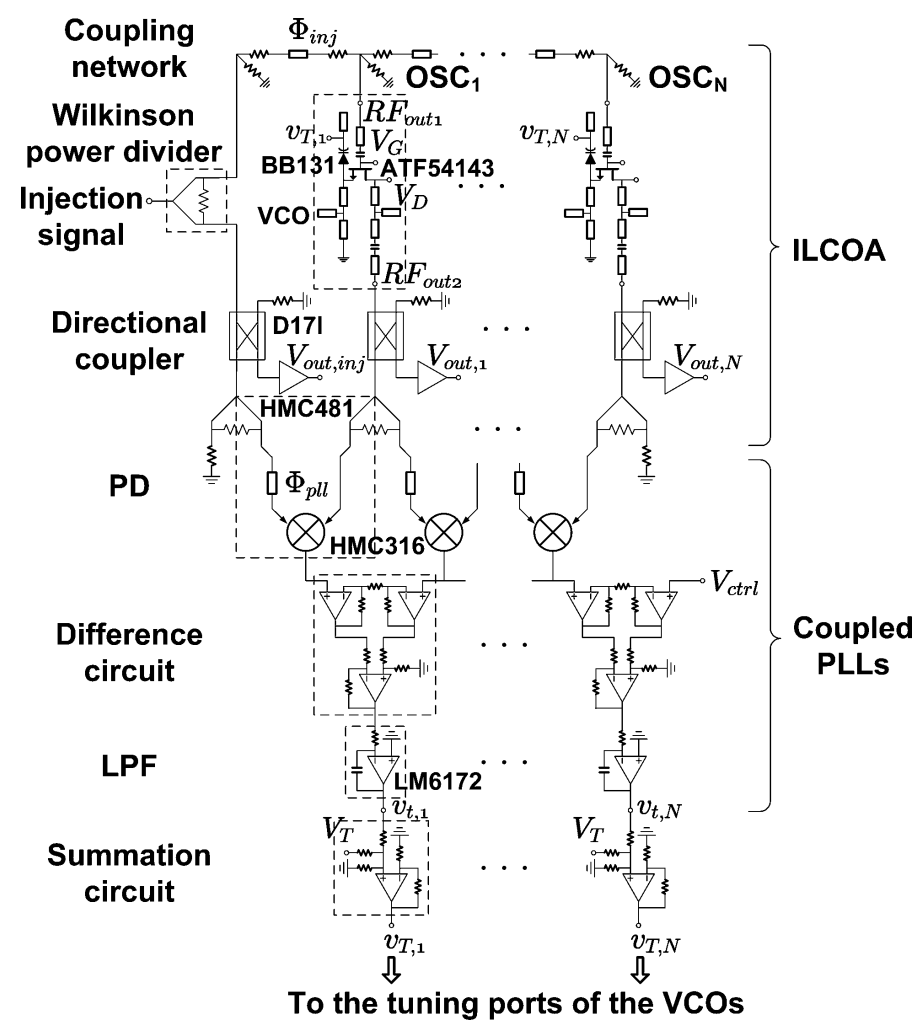

Fig. 2. Circuit schematic diagram of an $N$-element ILCOA integrated with coupled type-II PLLs.

Results of a single-element unit are described. Then, the control of the uniform phase progression and reduction of phase errors are verified experimentally with a three-element ILCOA. Finally, by loading this three-element ILCOA with rectangular patch antennas, the radiation characteristics of this antenna array are measured to demonstrate the beam-steering capability over the operation frequency band.

\section{A. Circuit Implementation}

Fig. 2 illustrates the circuit schematic diagram of an $N$-element ILCOA integrated with coupled type-II PLLs. The design frequency is $2.7 \mathrm{GHz}$. The array components are fabricated on FR4 substrate with $1.6 \mathrm{~mm}$ thickness. The injection signal is applied to the upper left Wilkinson power divider to be separated into two signals. One is applied to a resistive coupling network to lock the oscillator array, while the other is fed to the connected PD through a Mini-Circuits D17I directional coupler and another Wilkinson power divider. The signal $V_{\text {out,inj }}$ at the output port of a Hittite HMC481 amplifier can be measured by an Agilent $54855 \mathrm{~A}$ oscilloscope for monitoring the injection signal or loaded by an antenna for radiation.

Each of the oscillators shown in Fig. 2 consists of a voltagecontrolled oscillator (VCO), a Mini-Circuit D17I directional coupler, a Hittite HMC481 amplifier, and a Wilkinson power divider. The VCO has two output ports, $\mathrm{RF}_{\text {out1 }}$ and $\mathrm{RF}_{\text {out2 }}$. $\mathrm{RF}_{\text {out } 1}$ is connected to a resistive coupling network for the mutual coupling of the oscillator array. The directional coupler and power divider separate the signal at $\mathrm{RF}_{\text {out2 } 2}$ into three signals. The signal $V_{\text {out }, i}$ at the output port of each HMC481 amplifier 


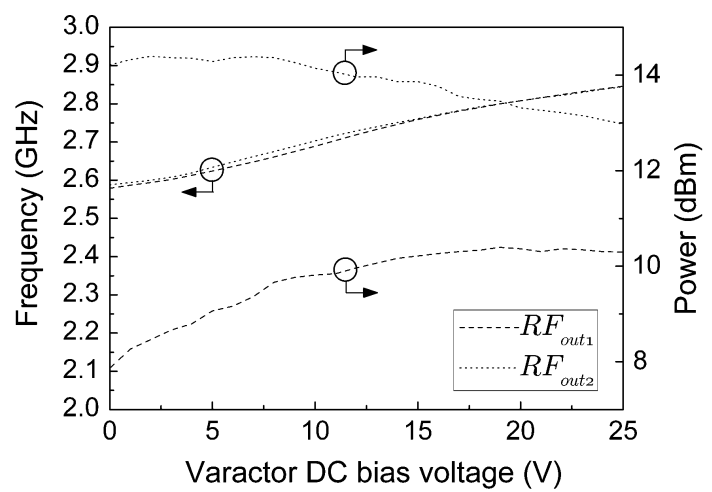

(a)

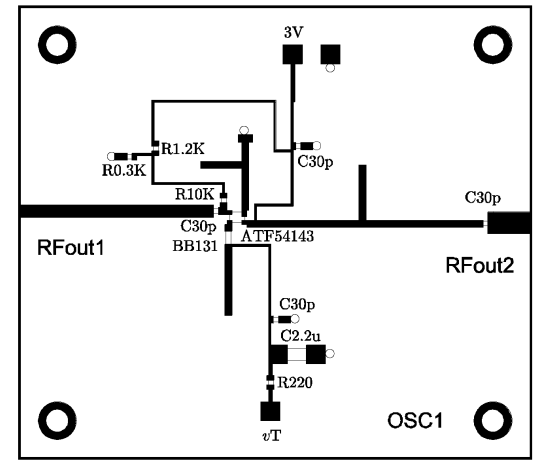

(b)

Fig. 3. (a) Measured results of VCO output power levels and tuning frequency and (b) VCO board layout.

can be measured by an Agilent 54855A oscilloscope or radiated through a connected antenna, while the other two signals at the output ports of the power divider are applied to the connected PDs for phase comparison.

The VCO circuit uses an Agilent ATF-54143 low noise enhancement mode pHEMT with series feedback at the transistor source terminal to provide negative resistance for oscillation and a Philips BB-131 VHF varactor to tune the free-running frequency. Fig. 3(a) shows the VCO tuning range and power levels at the two output ports, which gives $K_{v} \simeq 11 \mathrm{MHz} / \mathrm{V}$ in the linear region. Fig. 3(b) shows the VCO board layout where the pads marked with small circles are vias to the ground.

The PD uses a Hittite HMC316 double-balanced mixer to measure the phase difference between the output signals of two successive oscillators. As one applies input signals with the same power level to the PD RF and LO ports $(13 \mathrm{dBm}$ at 2.7 $\mathrm{GHz}$ in this design) and varies their phase difference $\Delta \theta$, the measured PD DC output voltage $V_{\text {pd,out }}$ shown in Fig. 4 gives the curve fitting relation as

$$
V_{\mathrm{pd}, \mathrm{out}}=0.72 \sin \left(\Delta \theta-1.4^{\circ}\right)
$$

with $K_{\mathrm{pd}} \simeq 0.72 \mathrm{~V}$.

The resistive coupling network is implemented with microstrip transmission lines terminated with resistors, and $\epsilon$ and $\Phi_{\text {inj }}$ are set to be 0.1 and $-2 \pi$, respectively. With the use of

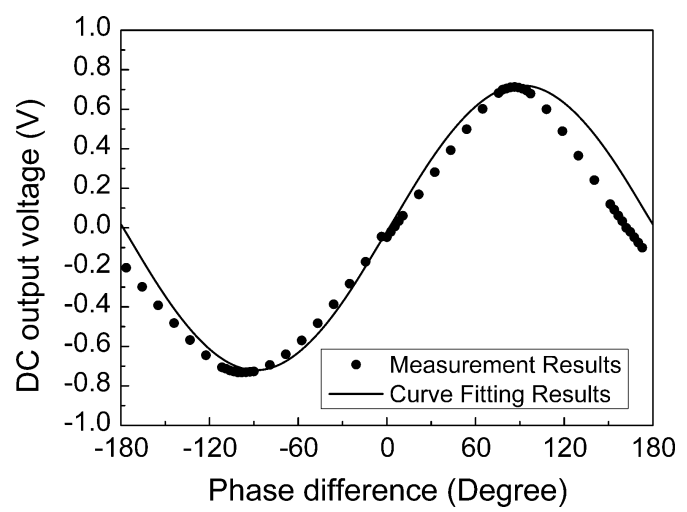

Fig. 4. Measured results of PD DC output voltage for different values of the phase difference.

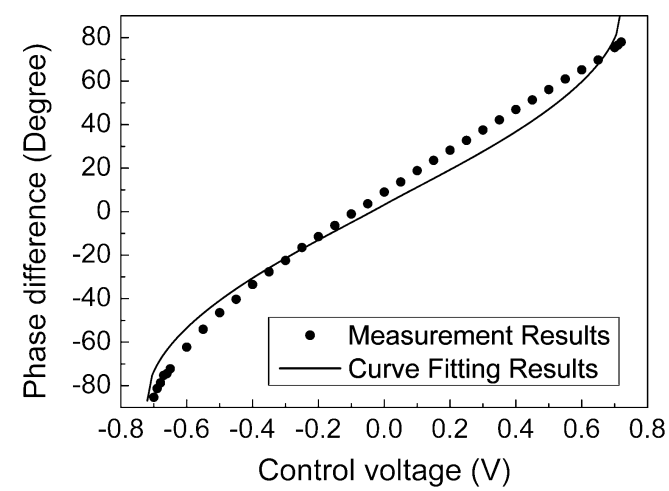

Fig. 5. Measured results of the phase difference of an ILO integrated with a type-II PLL.

National Semiconductor LM6172 high-speed voltage feedback amplifiers, the voltage difference circuit is realized as an instrumentation amplifier [24], and the LPF is implemented as an inverting integrator. Although the loop filter of the inverting integrator would inevitably put a PLL on the verge of instability, the proposed architecture is stable because of COA coupling. In other words, COA coupling influences stability and simplifies the loop filter design. In addition, the summation circuit is used to add the varactor signal voltage $v_{t}$ to the varactor DC bias voltage $V_{T}(12.5 \mathrm{~V}$ in this design) to give the varactor tuning voltage $v_{T}$ applied to the $\mathrm{VCO}$ varactor with a range from 0.04 $\mathrm{V}$ to $23.6 \mathrm{~V}$.

\section{B. Measurement of a Single-Element Unit}

The signals at the output ports of the HMC481 amplifiers fed by the VCO and the injection signal for the single-element unit are measured with an Agilent 54855A oscilloscope. The measured values of $\Delta \theta$ at the different control voltages shown in Fig. 5 give the curve fitting relation as

$$
\Delta \theta=\sin ^{-1}\left(\frac{V_{\mathrm{ctrl}}}{K_{\mathrm{pd}}}\right)+3.1^{\circ} .
$$

The small discrepancy between the phase shifts of $1.4^{\circ}$ and $3.1^{\circ}$ for (9) and (10) may be caused by the offset voltages of oper- 


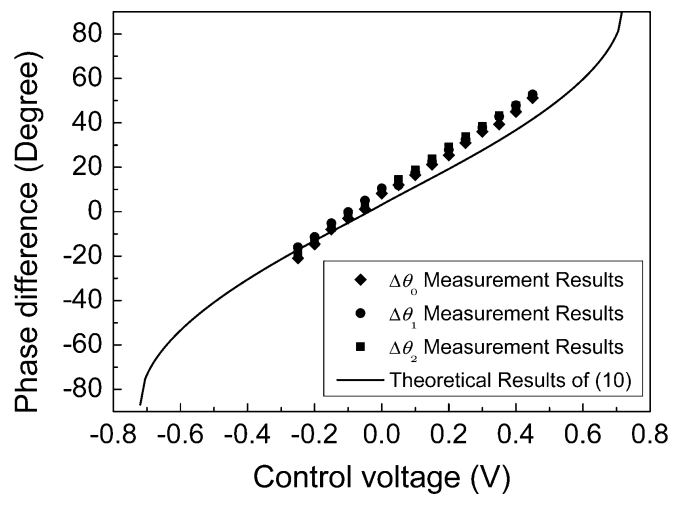

Fig. 6. Measured results of the uniform phase progression of a three-element ILCOA integrated with coupled type-II PLLs.

TABLE I

Measured Values of Phase Differences of a THREe-ElEment ILCOA INTEGRATED WITH COUPLED TYPE-II PLLS

\begin{tabular}{crrr}
\hline & \multicolumn{3}{c}{$\begin{array}{c}\text { Phase progression } \\
\text { (degree) }\end{array}$} \\
\cline { 2 - 4 }$V_{\text {ctrl }}$ & $\Delta \theta_{0}$ & $\Delta \theta_{1}$ & $\Delta \theta_{2}$ \\
\hline-0.25 & -21.0 & -16.0 & -18.6 \\
-0.20 & -14.7 & -11.4 & -12.3 \\
-0.15 & -8.0 & -5.2 & -7.1 \\
-0.10 & -3.0 & -0.2 & -1.9 \\
-0.05 & 1.2 & 5.0 & 3.8 \\
0.00 & 8.3 & 10.5 & 9.1 \\
0.05 & 12.0 & 11.8 & 14.5 \\
0.10 & 16.5 & 17.6 & 18.8 \\
0.15 & 21.2 & 22.7 & 23.9 \\
0.20 & 25.4 & 27.9 & 29.1 \\
0.25 & 31.0 & 32.5 & 33.9 \\
0.30 & 36.0 & 37.9 & 38.4 \\
0.35 & 39.3 & 42.9 & 43.2 \\
0.40 & 45.0 & 47.9 & 47.7 \\
0.45 & 51.3 & 52.8 & 52.3 \\
\hline
\end{tabular}

ational amplifiers. The range of the phase difference is shown about $\pm 90^{\circ}$, which is in good agreement with the theoretical result.

The locking range, over which the unit maintains synchronization but it may not necessarily acquire locking, is measured under the conditions where $V_{T}$ and $V_{\text {ctrl }}$ are respectively set at $12.5 \mathrm{~V}$ and $0 \mathrm{~V}$ and the power level of the external injection signal is $16.5 \mathrm{dBm}$. The measured locking range is about 400 $\mathrm{MHz}$ and approximately equals to the sum of the tuning range and the injection locking range of a $\mathrm{VCO}$, which can be predicted from (1).

\section{Measurement of a Three-Element ILCOA}

The signals at the output ports of the HMC481 amplifiers for a three-element ILCOA are measured with an Agilent 54855A oscilloscope. The measured values of $\Delta \theta_{i}$ between the output signals of successive oscillators at the different control voltages are shown in Fig. 6, where $\Delta \theta_{0}=\theta_{\text {inj }}-\theta_{1}$ and $\Delta \theta_{i}=\theta_{i}-\theta_{i+1}$ are defined in (2) and (3). They are consistent with the predicted values based on (10). Table I quantitatively shows the measured phase differences at $2.7 \mathrm{GHz}$ to give detailed information about

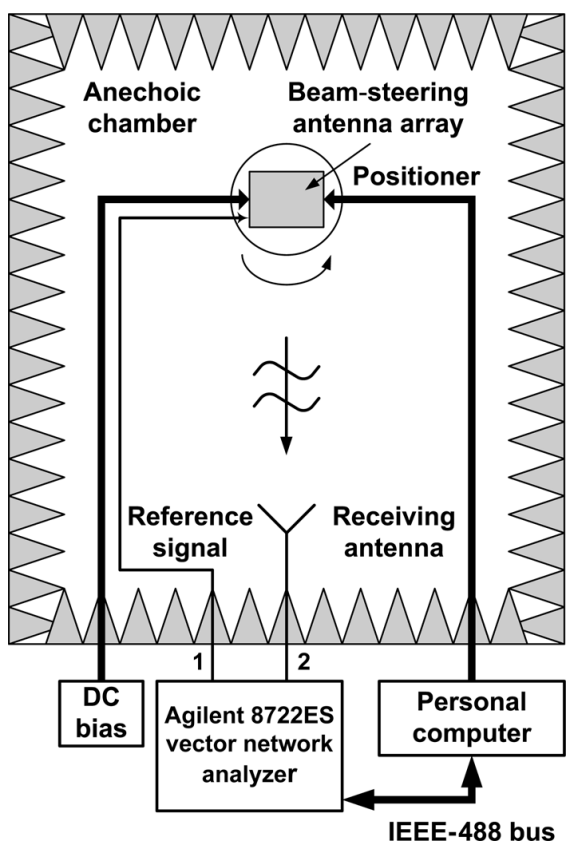

Fig. 7. Experimental arrangement for radiation pattern measurement.

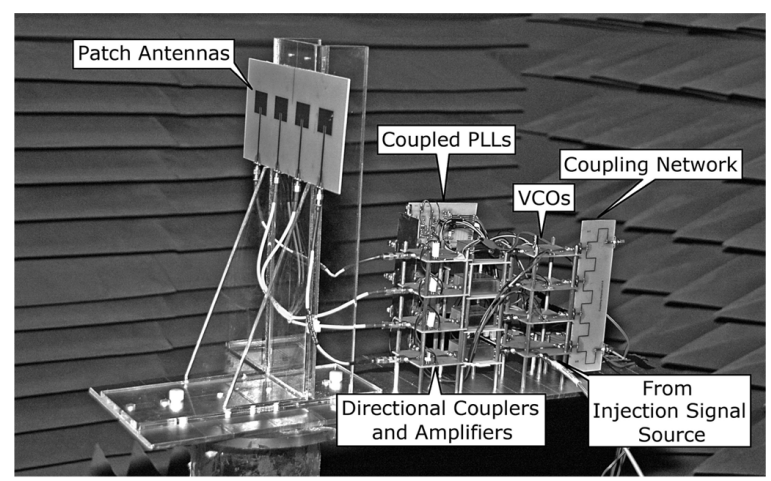

(a)

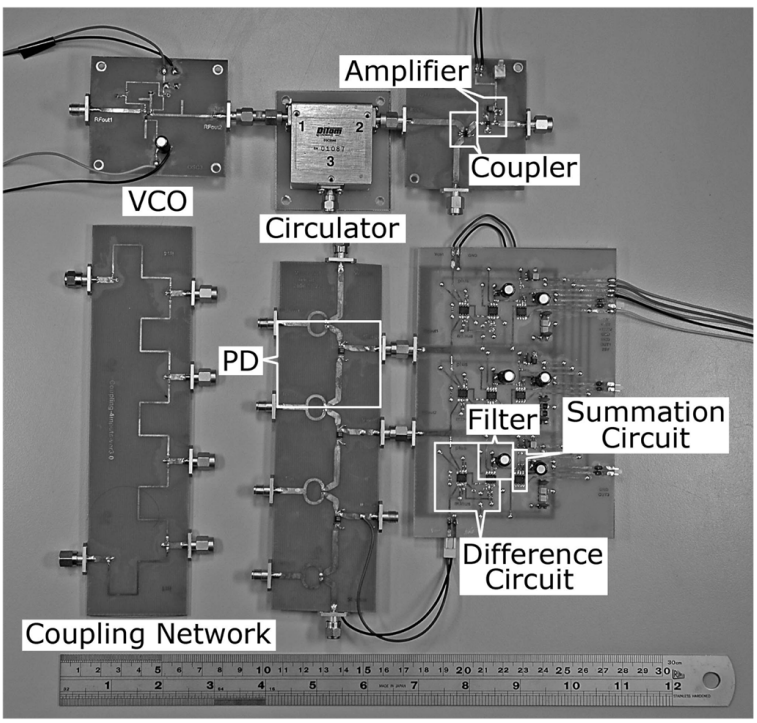

(b)

Fig. 8. Photographs of (a) the three-element beam-steering antenna array and (b) the related components.

phase control. The measured phase errors are shown to be less than $5^{\circ}$ for all the values of $V_{\mathrm{ctrl}}$. The phase errors are mainly 


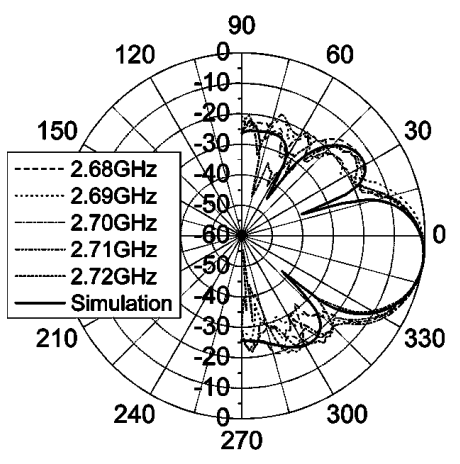

(a)

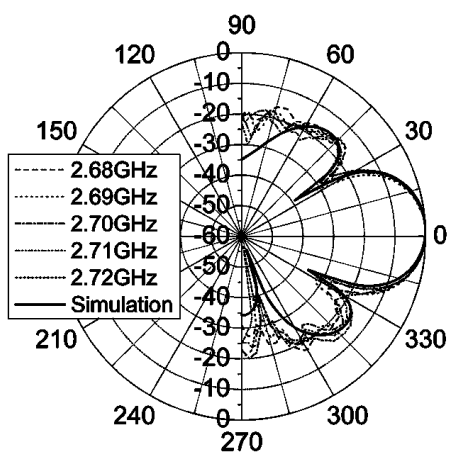

(d)

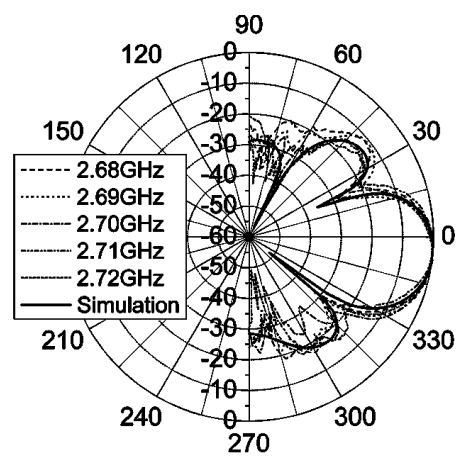

(b)

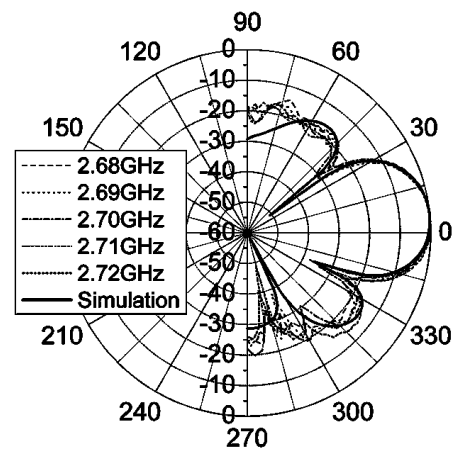

(e)

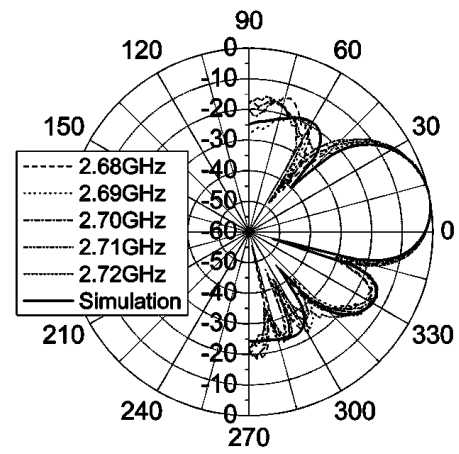

(g)

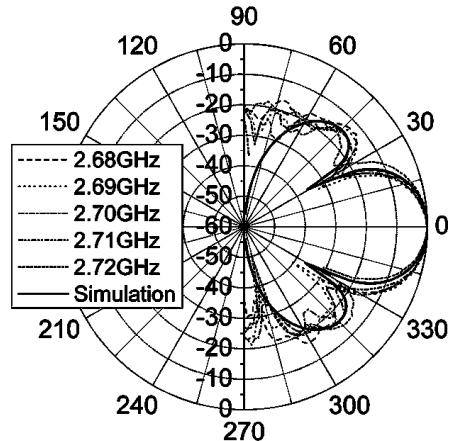

(c)

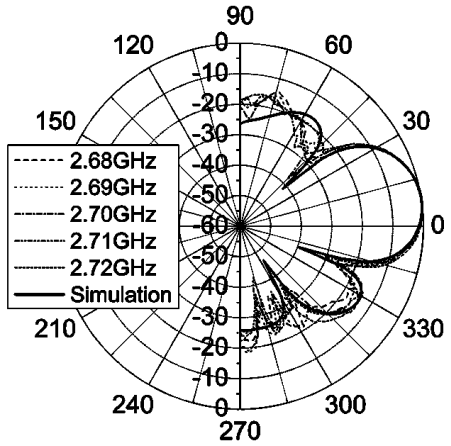

(f)

Fig. 9. Measured and simulated radiation patterns of a three-element beam-steering antenna array at the control voltages of (a) $-0.2 \mathrm{~V}$, (b) $-0.1 \mathrm{~V}$, (c) $0 \mathrm{~V}$, (d) $0.1 \mathrm{~V}$, (e) $0.2 \mathrm{~V}$, (f) $0.3 \mathrm{~V}$, and (g) $0.4 \mathrm{~V}$ over the operation frequency band from $2.68-2.72 \mathrm{GHz}$.

caused by noises and circuit mismatches arising from fabrication inconsistencies, especially by the mismatch among the PDs. The range of the uniform phase progression is from $-16^{\circ}$ to $52^{\circ}$. It demonstrates that the value of the uniform phase progression can be determined by an accurate and convenient approach. The range becomes smaller than that of a single-element unit because the difference between the injection signal frequency and the common frequency of the oscillator array is limited to the locking range divided by the number of oscillators in the array [25]. However, using frequency multipliers to extend its range [26] could overcome this drawback.

The locking range is measured under the same conditions as those of the single-element unit and gives around $250 \mathrm{MHz}$. The narrower locking range is due to the variations in the tuning ranges of the three VCOs and the limited range of the differ- ence between the injection signal frequency and the common frequency of the oscillator array.

Fig. 7 shows the experimental arrangement for measuring the radiation characteristics of the developed beam-steering antenna array. The injection signal is from an Agilent 8722ES vector network analyzer. The beam-steering antenna array shown in Fig. 8(a) is located on a computer-controlled positioner. Within the antenna array the right-end patch antenna is connected at $V_{\text {out,inj }}$ port as shown in Fig. 2 and fed by the external injection signal through a Wilkinson power divider, a D17I directional coupler, and an HMC481 amplifier, while the other three ones are connected at $V_{\text {out }, 1}$ to $V_{\text {out }, 3}$ ports to the respective VCOs. Fig. 8(b) shows the typical components. In the measurement, a metallic box covers the circuits at the back of patch antennas to provide proper electromagnetic shielding. The radiation pattern 
is then measured in the far field. A personal computer is adopted to operate this measurement system via an IEEE-488 bus to perform positioner rotation, spectrum monitoring, and data acquisition.

The measured and simulated results for different control voltages ranging from $-0.2 \mathrm{~V}$ to $0.4 \mathrm{~V}$ with $0.1 \mathrm{~V}$ spacing are shown in Fig. 9. The resulting scanning angles of the main beam are shown at $-9^{\circ},-7.2^{\circ}, 0^{\circ}, 3.6^{\circ}, 5.4^{\circ}, 9^{\circ}$, and $12.6^{\circ}$, respectively. For each measurement, the injection signal frequencies are given at five different frequencies of 2.68 , $2.69,2.70,2.71$, and $2.72 \mathrm{GHz}$. The measured results are shown to be in agreement with the simulated results. The results show that the direction of the main beam is controlled through a single control voltage. In addition, the beam-steering phased array works well and gives similar radiation patterns over a frequency band ranging from $2.68-2.72 \mathrm{GHz}$ and its operation frequency is determined by the injection signal. However, controlling the beam-scanning angle and the operation frequency of a conventional COA-based antenna array may require significant time and efforts when fluctuations in the free-running frequencies and amplitudes of the oscillators are considered. The experimental results demonstrate that the developed ILCOA-based antenna array integrated with coupled type-II PLLs can self-tune the free-running frequency distribution of the oscillator array for beam-steering applications in a cost-effective way.

\section{CONCLUSION}

In this paper, an ILCOA circuit configuration is presented with analysis and experimental results. The developed oscillator array and the beam-steering antenna array are measured and their performances are consistent with the simulated results. With the use of the coupled type-II PLLs and an external injection signal, the developed oscillator array can offer its output signals with a uniform progressive phase distribution through a single control signal and significantly reduce the phase errors caused by fluctuations in the amplitudes and free-running frequencies of the oscillators.

In general, there are two types of coupling for a COA, unilateral coupling and bilateral coupling. For unilateral coupling each element is injection locked to the preceding element in the array, whereas for bilateral coupling each element is mutually coupled to its neighboring elements. Similarly, for coupled PLLs the architecture with the control voltage passed from element to element through the difference circuit corresponds to a COA with bilateral coupling, while the architecture with the control voltage globally distributed on a single bus corresponds to a COA with unilateral coupling. Although the ILCOA presented in this paper utilizes bilaterally coupled PLLs as shown in Fig. 1, both PLL coupling approaches can work well and make no difference for the purpose of phase control since the control signal is a DC voltage. Comparison on the array performance with the use of these two coupling approaches is under study.

There are few more remarks on the prospects of the ILCOA given in the following. First, as the phase distribution is more precisely set by coupled type-II PLLs than by a COA, another promising approach may use coupled type-II PLLs with independent oscillators and an external reference signal. The loop filter of each element then becomes the design issue. Second, each element of a COA integrated with coupled PLLs needs more cost and larger space due to PLL circuits. The approach of an ILCOA integrated with coupled type-II PLLs, however, provides not only precise phase control but also phase monitoring. The output voltages of the PDs can be digitized and processed with computer software as in [27], [28]. Thirdly, since the output frequency of the oscillator array is controlled by the injection signal, it has the potential for the communication system of multiple-access technique, such as frequency-division multiple access or code-division multiple access. With a modulated injection signal, it is also suitable for the frequency modulation and phase modulation applications. In [29], the study proposes another approach in an actual communication system, which relies on injection locking for distributing and phase locking the carrier signal and uses digital-to-analog controlled mixers for beamforming and data modulation. Finally, by using multilayer circuit board, the developed approach could be implemented into a two-dimensional array design for communication or radar system applications.

\section{APPENDIX \\ STABILITY ANALYSIS}

The stability of the solution to nonlinear equations can be analyzed by linearizing the equations around their equilibrium points. In this Appendix, the stability analysis of (1) is given in the following. For simplicity, assume $A_{i}=A$ and let $\hat{\theta}_{i}$ be a solution of (1). As it is perturbed by having $\theta_{i}=\hat{\theta}_{i}+\delta \theta_{i}$ with small perturbation $\delta \theta_{i}$, (1) becomes

$$
\begin{aligned}
\frac{d}{d t} \delta \theta_{\mathrm{inj}}= & \delta \omega_{\mathrm{inj}} \\
\frac{d}{d t} \delta \theta_{i}= & K_{v} K_{\mathrm{pd}}\left[\sin \left(\Delta \hat{\theta}_{i-1}+\Phi_{\mathrm{pll}}\right)\right. \\
& \left.\times \delta \Delta \theta_{i-1}-\sin \left(\Delta \hat{\theta}_{i}+\Phi_{\mathrm{pll}}\right) \delta \Delta \theta_{i}\right] * f(t) \\
& +\frac{\epsilon \omega_{\mathrm{fr}, i}}{2 Q_{i}}\left[\cos \left(\Delta \hat{\theta}_{i-1}+\Phi_{\mathrm{inj}}\right)\right. \\
& \left.\times \delta \Delta \theta_{i-1}-\cos \left(\Delta \hat{\theta}_{i}-\Phi_{\mathrm{inj}}\right) \delta \Delta \theta_{i}\right] \\
\frac{d}{d t} \delta \theta_{N}= & K_{v} K_{\mathrm{pd}} \sin \\
& \times\left(\Delta \hat{\theta}_{N-1}+\Phi_{\mathrm{pll}}\right) \delta \Delta \theta_{N-1} * f(t) \\
& +\frac{\epsilon \omega_{\mathrm{fr}, N}}{2 Q_{N}} \cos \left(\Delta \hat{\theta}_{N-1}+\Phi_{\mathrm{inj}}\right) \delta \Delta \theta_{N-1}
\end{aligned}
$$

where $i=1, \ldots, N-1 . \delta \omega_{\mathrm{inj}}$ stands for the frequency variation of the injection signal and $\delta \omega_{\text {inj }}=0$ if the injection signal is a continuous wave. Since the stability of the phase difference between the output signals of successive oscillators is of great concern, (A.1) can be cast in the form of relative phase. Now that the free-running frequencies $\omega_{\mathrm{fr}, i}$ must be close in value on the order of $\epsilon \omega_{\mathrm{fr}, i} / 2 Q_{i}$ for synchronization to occur [12], one can let $\epsilon \omega_{\mathrm{fr}, i} / 2 Q_{i}$ be the same for all $N$ oscillators, i.e., $\epsilon \omega_{\mathrm{fr}, i} / 2 Q_{i}=\epsilon \omega_{\mathrm{fr}} / 2 Q$ for $i=1, \ldots, N$. Letting

$$
\begin{aligned}
\delta \Delta \theta_{0} & =\delta \theta_{\mathrm{inj}}-\delta \theta_{1} \\
\delta \Delta \theta_{i} & =\delta \theta_{i}-\delta \theta_{i+1}
\end{aligned}
$$


and $f(t)=-u(t) / R C,(\mathrm{~A} .1)$ then is expressed as

$$
\begin{aligned}
\frac{d}{d t} \delta \Delta \theta_{0}= & \delta \omega_{\text {inj }} \\
& +\left(-C_{0}^{\mathrm{pll}} \delta \Delta \theta_{0}+C_{1}^{\mathrm{pll}} \delta \Delta \theta_{1}\right) * u(t) \\
& -C_{0, a}^{\mathrm{inj}} \delta \Delta \theta_{0}+C_{1, b}^{\mathrm{inj}} \delta \Delta \theta_{1} \\
\frac{d}{d t} \delta \Delta \theta_{i}= & \left(C_{i-1}^{\mathrm{pll}} \delta \Delta \theta_{i-1}-2 C_{i}^{\mathrm{pll}} \delta \Delta \theta_{i}\right. \\
& \left.+C_{i+1}^{\mathrm{pll}} \delta \Delta \theta_{i+1}\right) * u(t) \\
& +C_{i-1, a}^{\mathrm{inj}} \delta \Delta \theta_{i-1}-C_{i, t}^{\mathrm{inj}} \delta \Delta \theta_{i}+C_{i+1, b}^{\mathrm{inj}} \delta \Delta \theta_{i+1}
\end{aligned}
$$

where

$$
\begin{aligned}
C_{i}^{\mathrm{pll}} & =-\frac{K_{v} K_{\mathrm{pd}}}{R C} \sin \left(\Delta \hat{\theta}_{i}+\Phi_{\mathrm{pll}}\right) \\
C_{i, a}^{\mathrm{inj}} & =\frac{\epsilon \omega_{\mathrm{fr}}}{2 Q} \cos \left(\Delta \hat{\theta}_{i}+\Phi_{\mathrm{inj}}\right) \\
C_{i, b}^{\mathrm{inj}} & =\frac{\epsilon \omega_{\mathrm{fr}}}{2 Q} \cos \left(\Delta \hat{\theta}_{i}-\Phi_{\mathrm{inj}}\right) \\
C_{i, t}^{\mathrm{inj}} & =C_{i, a}^{\mathrm{inj}}+C_{i, b}^{\mathrm{inj}} .
\end{aligned}
$$

Note that $C_{N}^{\mathrm{pll}}=C_{N, b}^{\mathrm{inj}}=0$.

Let

$$
\begin{aligned}
\frac{d}{d t} \delta \Delta \theta_{0,1} & =-C_{0}^{\mathrm{pll}} \delta \Delta \theta_{0}+C_{1}^{\mathrm{pll}} \delta \Delta \theta_{1} \\
\frac{d}{d t} \delta \Delta \theta_{i, 1} & =C_{i-1}^{\mathrm{pll}} \delta \Delta \theta_{i-1}-2 C_{i}^{\mathrm{pll}} \delta \Delta \theta_{i}+C_{i+1}^{\mathrm{pll}} \delta \Delta \theta_{i+1}
\end{aligned}
$$

and the state variable vector be

$$
\begin{aligned}
\bar{x}=\left[\begin{array}{ll}
\delta \Delta \theta_{0} \quad \delta \Delta & \theta_{0,1} \quad \delta \Delta \theta_{1} \\
& \times \delta \Delta \theta_{1,1} \cdots \delta \Delta \theta_{N-1} \quad \delta \Delta \theta_{N-1,1}
\end{array}\right]^{T} .
\end{aligned}
$$

The state-space representation of (A.4) then is expressed as

$$
\begin{aligned}
\frac{d}{d t} \bar{x} & =\mathbf{A} \bar{x}+\mathbf{B} \bar{u} \\
\bar{y} & =\mathbf{C} \bar{x}
\end{aligned}
$$

where $\bar{y}$ is the output vector and $\bar{u}=\left[\delta \omega_{\text {inj }}\right]$ is the input scalar. $\mathbf{A}, \mathbf{B}$, and $\mathbf{C}$ are $2 N \times 2 N, 2 N \times 1$, and $N \times 2 N$ matrices, respectively, and are given as (A.14), (A.15), and (A.16), shown at the bottom of the page.

For the developed oscillator array, the phase distribution is stable with respect to small perturbation if the perturbation dies out with time. That is all the eigenvalues of $\mathbf{A}$ for this forced system must have negative real parts [7], [12], [30]. With $\Phi_{\text {inj }}=-2 \pi, \Phi_{\text {pll }}=-\pi / 2, K_{v}=11 \mathrm{MHz} / \mathrm{V}, K_{\mathrm{pd}}=0.72 \mathrm{~V}$, $\epsilon \omega_{\mathrm{fr}} / 2 Q=532.8 \mathrm{Mrad} / \mathrm{sec}, R=1 \mathrm{~K} \Omega$, and $C=22 \mathrm{uF}$, the eigenvalues of $\mathbf{A}$ for each mode given in (6) are calculated for the developed three-element oscillator array. Only the phase distribution with uniform phase progressions meets the stability requirement and thus is the stable solution of (1). The stable range of phase progression is limited to $\pm 90^{\circ}$.

Nevertheless, stability depends on phase gradient and the number of array elements [7]. Furthermore, noises and non-identical array elements are encountered in practical arrays. Therefore, the measured stable range would be smaller than the predicted value and stability might not be guaranteed as the number of array elements increases. Linear stability

$$
\begin{aligned}
& \mathbf{A}=\left[\begin{array}{ccccccccccc}
-C_{0, a}^{\text {inj }} & 1 & C_{1, b}^{\text {inj }} & 0 & 0 & 0 & \ldots & 0 & 0 & 0 & 0 \\
-C_{0}^{\text {pll }} & 0 & C_{1}^{\text {pll }} & 0 & 0 & 0 & \ldots & 0 & 0 & 0 & 0 \\
C_{0, a}^{\text {inj }} & 0 & -C_{1, t}^{\text {inj }} & 1 & C_{2, b}^{\text {inj }} & 0 & \ldots & 0 & 0 & 0 & 0 \\
C_{0}^{\text {pll }} & 0 & -2 C_{1}^{\text {pll }} & 0 & C_{2}^{\text {pll }} & 0 & \ldots & 0 & 0 & 0 & 0 \\
0 & 0 & C_{1, a}^{\text {inj }} & 0 & -C_{2, t}^{\text {inj }} & 1 & \ldots & 0 & 0 & 0 & 0 \\
0 & 0 & C_{1}^{\text {pll }} & 0 & -2 C_{2}^{\text {pll }} & 0 & \ldots & 0 & 0 & 0 & 0 \\
\vdots & \vdots & \vdots & \vdots & \vdots & \vdots & \vdots: \vdots & \vdots & \vdots & \vdots & \vdots \\
0 & 0 & 0 & 0 & 0 & 0 & \ldots & -C_{N-2, t}^{\text {inj }} & 1 & C_{N-1, b}^{\text {inj }} & 0 \\
0 & 0 & 0 & 0 & 0 & 0 & \ldots & -2 C_{N-2}^{\text {pll }} & 0 & C_{N-1}^{\text {pll }} & 0 \\
0 & 0 & 0 & 0 & 0 & 0 & \ldots & C_{N-2, a}^{\text {inj }} & 0 & -C_{N-1, t}^{\text {inj }} & 1 \\
0 & 0 & 0 & 0 & 0 & 0 & \ldots & C_{N-2}^{\text {pll }} & 0 & -2 C_{N-1}^{\text {pll }} & 0
\end{array}\right] \\
& \mathbf{B}=\left[\begin{array}{llll}
1 & 0 & \cdots & 0
\end{array}\right]^{T} \\
& \mathbf{C}=\left[\begin{array}{ccccccccccc}
1 & 0 & 0 & 0 & 0 & 0 & \cdots & 0 & 0 & 0 & 0 \\
0 & 0 & 1 & 0 & 0 & 0 & \cdots & 0 & 0 & 0 & 0 \\
\vdots & \vdots & \vdots & \vdots & \vdots & \vdots & \vdots: \vdots & \vdots & \vdots & \vdots & \vdots \\
0 & 0 & 0 & 0 & 0 & 0 & \cdots & 1 & 0 & 0 & 0 \\
0 & 0 & 0 & 0 & 0 & 0 & \cdots & 0 & 0 & 1 & 0
\end{array}\right] .
\end{aligned}
$$


analysis, however, gives useful information about circuit parameters for initial design.

\section{ACKNOWLEDGMENT}

The authors would like to thank C. W. Hsu and S. N. Hsieh for their helpful discussions.

\section{REFERENCES}

[1] P. Liao and R. A. York, "A new phase-shifterless beam-scanning technique using arrays of coupled oscillators," IEEE Trans. Microw. Theory Tech., vol. 41, no. 10, pp. 1810-1815, Oct. 1993.

[2] R. J. Pogorzelski, P. F. Maccarini, and R. A. York, "A continuum modeling of the dynamics of coupled oscillator arrays for phase-shifterless beam scanning," IEEE Trans. Microw. Theory Tech., vol. 47, no. 4, pp. 463-470, Apr. 1999.

[3] J. A. Navarro and K. Chang, Integrated Active Antennas and Spatial Power Combining. New York: Wiley, 1996.

[4] S. T. Chew, T. K. Tong, M. C. Wu, and T. Itoh, "An active phased array with optical input and beam-scanning capability," IEEE Microw. Guided Wave Lett., vol. 4, no. 10, pp. 347-349, Oct. 1994.

[5] R. A. York and T. Itoh, "Injection- and phase-locking techniques for beam control," IEEE Trans. Microw. Theory Tech., vol. 46, no. 11, pp. 1920-1929, Nov. 1998.

[6] S. C. Yen and T. H. Chu, "A beam-scanning and polarization-agile antenna array using mutually coupled oscillating doublers," IEEE Trans. Antennas Propag., vol. 53, no. 12, pp. 4051-4057, Dec. 2005.

[7] T. Heath, "Beam steering of nonlinear oscillator arrays through manipulation of coupling phases," IEEE Trans. Antennas Propag., vol. 52, no. 7, pp. 1833-1842, Jul. 2004.

[8] K. D. Stephan, "Inter-injection-locked oscillators for power combining and phased arrays," IEEE Trans. Microw. Theory Tech., vol. 34, no. 10, pp. 1017-1025, Oct. 1986.

[9] R. Adler, "A study of locking phenomena in oscillators," Proc. IRE, vol. 34, pp. 351-357, Jun. 1946.

[10] K. Kurokawa, "Injection locking of microwave solid-state oscillators," Proc. IEEE, vol. 61, no. 10, pp. 1386-1410, Oct. 1973.

[11] C. C. Huang and T. H. Chu, "Analysis of MESFET injection-locked oscillators in fundamental mode of operation," IEEE Trans. Microw. Theory Tech., vol. 42, no. 10, pp. 1851-1857, Oct. 1994.

[12] R. A. York, "Nonlinear analysis of phase relationships in quasi-optical oscillator arrays," IEEE Trans. Microw. Theory Tech., vol. 41, no. 10, pp. 1799-1809, Oct. 1993.

[13] R. A. York, P. Liao, and J. J. Lynch, "Oscillator array dynamics with broadband N-port coupling networks," IEEE Trans. Microw. Theory Tech., vol. 42, no. 11, pp. 2040-2045, Nov. 1994.

[14] H. C. Chang, E. S. Shapiro, and R. A. York, "Influence of the oscillator equivalent circuit on the stable modes of parallel-coupled oscillators," IEEE Trans. Microw. Theory Tech., vol. 45, no. 8, pp. 1232-1239, Aug. 1997.

[15] J. Shen and L. W. Pearson, "The phase error and beam-pointing error in coupled oscillator beam-steering array," IEEE Trans. Antennas Propag., vol. 53, no. 1, pp. 386-393, Jan. 2005.

[16] X. Wang and L. W. Pearson, "Design of coupled-oscillator arrays without a posteriori tuning," IEEE Trans. Microw. Theory Tech., vol. 53, no. 1, pp. 410-413, Jan. 2005.

[17] R. D. Martinez and R. C. Compton, "Electronic beamsteering of active arrays with phase-locked loop," IEEE Microw. Guided Wave Lett., vol. 4, no. 6, pp. 166-168, Jun. 1994.

[18] J. F. Buckwalter, T. H. Heath, and R. A. York, "Synchronization design of a coupled phase-locked loop," IEEE Trans. Microw. Theory Tech., vol. 51, no. 3, pp. 952-960, Mar. 2003.
[19] H. C. Chang, "Analysis of coupled phase-locked loops with independent oscillators for beam control active phased arrays," IEEE Trans. Microw. Theory Tech., vol. 52, no. 3, pp. 1059-1066, Mar. 2004.

[20] P. F. Maccarini, J. Buckwalter, and R. A. York, "Coupled phase-locked loop arrays for beam steering," in IEEE MTT-S Int. Microwave Symp. Digest, Jun. 2003, vol. 3, pp. 1689-1692.

[21] H. C. Chang, A. Borgioli, P. Yeh, and R. A. York, "Analysis of oscillators with external feedback loop for improved locking range and noise reduction," IEEE Trans. Microw. Theory Tech., vol. 47, no. 8, pp. 1535-1543, Aug. 1999.

[22] S. Nogi, J. Lin, and T. Itoh, "Mode analysis and stabilization of a spatial power combining array with strongly coupled oscillators," IEEE Trans. Microw. Theory Tech., vol. 41, no. 10, pp. 1827-1837, Oct. 1993.

[23] J. J. Lynch and R. A. York, "Synchronization of oscillators coupled through narrow-band networks," IEEE Trans. Microw. Theory Tech., vol. 49, no. 2, pp. 237-249, Feb. 2001.

[24] A. S. Sedra and K. C. Smith, Microelectronic Circuits. New York: Oxford Univ. Press, 2004.

[25] R. J. Pogorzelski, P. F. Maccarini, and R. A. York, "Continuum modeling of the dynamics of externally injection-locked coupled oscillator arrays," IEEE Trans. Microw. Theory Tech., vol. 47, no. 4, pp. 471-478, Apr. 1999.

[26] A. Alexanian, H. C. Chang, and R. A. York, "Enhanced scanning range of coupled oscillator arrays utilizing frequency multipliers," in IEEE AP-S Int. Symp. Digest, 1995, vol. 2, pp. 1308-1310.

[27] R. J. Pogorzelski, "Coupled oscillator based agile beam transmitters and receivers: A review of work at JPL," in Proc. IEEE Aerospace Conf., Mar. 2006, p. 1-14.

[28] T. Heath, R. R. Kerr, and G. D. Hopkins, "Nonlinear oscillator array antenna development at GTRI," in Proc. IEEE Aerospace Con., Mar. 2006, p. 1-19.

[29] J. F. Buckwalter, A. Babakhani, A. Komijani, and A. Hajimiri, "An integrated subharmonic coupled-oscillator scheme for a $60-\mathrm{GHz}$ phasedarray transmitter," IEEE Trans. Microw. Theory Tech., vol. 54, no. 12, pp. 4271-4280, Dec. 2006.

[30] R. C. Dorf and R. H. Bishop, Modern Control Systems. Menlo Park, CA: Addison-Wesley, 1998.

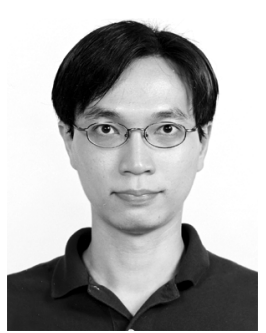

Sheng-Hong Yan (S’06) was born in Tainan, Taiwan, R.O.C., in 1977. He received the B.S. degree from in electrical engineering from National Taiwan University, Taipei, Taiwan, R.O.C., in 1999 and the M.S. and Ph.D. degrees from the Graduate Institute of Communication Engineering, National Taiwan University, in 2001 and 2008, respectively.

He is currently a Postdoctoral Research Fellow with the Department of Electrical Engineering, National Taiwan University. His research interests include microwave circuit design, phased array, and active antenna array techniques.

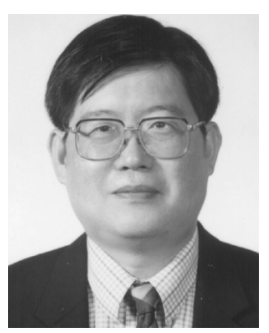

Tah-Hsiung Chu (M'87) received the B.S. degree from the National Taiwan University, Taipei, Taiwan, R.O.C., in 1976, and the M.S. and Ph.D. degrees from the University of Pennsylvania, Philadelphia, in 1980 and 1983, respectively, all in electrical engineering.

From 1983 to 1986 , he was a Member of Technical Staff with the Microwave Technology Center, RCA David Sarnoff Research Center, Princeton, NJ. Since 1986, he has been on the faculty of the Department of Electrical Engineering, National Taiwan University, where he is now a Professor of Electrical Engineering. His research interests include microwave-imaging systems and techniques, microwave circuits and subsystems, microwave measurements, and calibration techniques. 